Теорія Ймовір. та Матем. Статист. Вип. 75, 2006
Theor. Probability and Math. Statist.

No. 75, 2007, Pages 51-64 S 0094-9000(08)00713-8

Article electronically published on January 23, 2008

\title{
MAXIMAL UPPER BOUNDS FOR THE MOMENTS OF STOCHASTIC INTEGRALS AND SOLUTIONS OF STOCHASTIC DIFFERENTIAL EQUATIONS WITH RESPECT TO FRACTIONAL BROWNIAN MOTION WITH HURST INDEX $H<1 / 2$. I
}

UDC 519.21

\author{
YU. V. KOZACHENKO AND YU. S. MISHURA
}

\begin{abstract}
Upper moment bounds and maximal upper moment bounds are obtained for Wiener integrals considered with respect to a fractional Brownian motion with Hurst index $H<1 / 2$. Maximal bounds are derived from new maximal inequalities for Gaussian random variables and stochastic processes.
\end{abstract}

\section{INTRODUCTION}

Maximal moment bounds for martingales and stochastic integrals have been known for a long time in the literature (see, for example, 1]). On the other hand, the theory of stochastic integration has been developing in parallel for the case where the integrators are not semimartingales, say for a fractional Brownian motion. The general definition and main properties of Wiener integrals with respect to fractional Brownian motion with Hurst index $H \in(0,1 / 2)$, that is, integrals with nonrandom integrands, are given in the dissertation [2. Generally speaking, these properties are different for the cases of $H \in(0,1 / 2)$ and of $H \in(1 / 2,1)$ (note that the fractional Brownian motion $B^{H}=W$ is a Wiener process if $H=1 / 2$ ). Upper moment inequalities for Wiener integrals with respect to fractional Brownian motions with $H \in(1 / 2,1)$ are obtained in 3 . These bounds are generalized in 4 to the case of integrals with a random upper integration limit. Lower bounds are also obtained in 4. These bounds are used in [4] to estimate the index $H \in(0,1 / 2)$ with the help of the Sudakov inequality. In this paper, we obtain upper moment bounds for Wiener integrals with respect to fractional Brownian motion with $H \in(0,1 / 2)$.

The paper is organized as follows. Two cases of integrands are considered in Section 2, namely the case where the support of $f$ is $\mathbb{R}$ and the case where the support of $f$ is finite. New maximal inequalities for Gaussian random variables and stochastic processes are included in Section 3. We establish maximal moment inequalities for Wiener integrals with respect to fractional Brownian motion with $H \in(0,1 / 2)$ in Section 4 by applying the results obtained in Sections 2 and 3.

2000 Mathematics Subject Classification. Primary 60G15, 60H05.

Key words and phrases. Fractional Brownian motion, Wiener integral, moment inequalities, Gaussian stochastic processes.

This work is partially supported by the NATO grant PST.CLG.980408. 


\section{AN UPPER BOUND FOR MOMENTS OF WIENER INTEGRALS With RESPECT TO FRACTIONAL BROWNIAN MOTION WITH $H \in(0,1 / 2)$}

Let $B^{H}=\left\{B_{t}^{H}, \mathcal{F}_{t}, t \in \mathbb{R}\right\}$ be a fractional Brownian motion with Hurst index $H \in$ $(0,1 / 2)$; that is, $B^{H}$ is a Gaussian process such that $B_{0}^{H}=0, \mathrm{E} B_{t}^{H}=0$, and

$$
\mathrm{E} B_{t}^{H} B_{s}^{H}=\frac{1}{2}\left(|t|^{2 H}+|s|^{2 H}-|t-s|^{2 H}\right) .
$$

In what follows we consider a continuous modification of this process. Consider the operator $M_{-}^{H}$ such that $\left(M_{-}^{H} f\right)(x)=C_{H}^{(1)}\left(I_{-}^{\alpha} f\right)(x)$, where

$$
\alpha=H-1 / 2, \quad C_{H}^{(1)}=\left(\frac{2 H \Gamma(\alpha+1) \Gamma(1-\alpha)}{\Gamma(1+2 \alpha)}\right)^{1 / 2},
$$

and $I_{-}^{\alpha}$ is the operator of fractional differentiation, that is,

$$
\left(I_{-}^{\alpha} f\right)(x)=-\frac{1}{\Gamma(\alpha+1)} \frac{d}{d x} \int_{x}^{\infty} f(t)(t-x)^{\alpha} d t .
$$

We also consider the Wiener integral of the function $f$ with respect to the fractional Brownian motion $B_{t}^{H}$. According to the definition given in [2], the Wiener integral is a random variable

$$
\int_{\mathbb{R}} f(t) d B_{t}^{H}:=\int_{\mathbb{R}}\left(M_{-}^{H} f\right)(t) d W_{t},
$$

where $\left\{W_{t}, \mathcal{F}_{t}, t \in \mathbb{R}\right\}$ is a Wiener process for which the Mandelbrot-van Ness representation holds for $B^{H}$, that is, $B_{t}^{H}=\int_{R}\left(M_{-}^{H} 1_{(0, t)}\right)(s) d W_{s}$. The integral $\int_{\mathbb{R}} f(t) d B_{t}^{H}$ is well defined for functions

$$
f \in L_{H}^{2}(\mathbb{R}):=\left\{f: \mathbb{R} \rightarrow \mathbb{R} \mid \int_{\mathbb{R}}\left(\left(M_{-}^{H} f\right)(t)\right)^{2} d t<\infty\right\}
$$

whose norm is given by

$$
\|f\|_{L_{H}^{2}(\mathbb{R})}=\left(\int_{\mathbb{R}}\left(\left(M_{-}^{H} f\right)(t)\right)^{2} d t\right)^{1 / 2}
$$

(a) First we consider the case of functions $f$ with a general support (that is, we do not assume that the support is finite). Let $f \in C^{1}(\mathbb{R})$ and suppose

1) there are some numbers $c_{0}>0$ and $\varepsilon>0$ such that for all $|x|>1$,

$$
|f(x)|+\left|f^{\prime}(x)\right| \leq c_{0}|x|^{\alpha-1-\varepsilon} .
$$

By $W_{2}^{2}(\mathbb{R})$ we denote the standard Sobolev space, that is,

$$
W_{2}^{2}(\mathbb{R})=\left\{f: \mathbb{R} \rightarrow \mathbb{R} \mid\|f\|_{L_{2}(\mathbb{R})}+\left\|f^{\prime}\right\|_{L_{2}(\mathbb{R})}<\infty\right\} .
$$

Theorem 2.1. Let $f \in C^{1}(\mathbb{R}) \cap W_{2}^{2}(\mathbb{R})$ and let condition 1) hold. Then $f \in L_{H}^{2}(\mathbb{R})$ and there exists a constant $C_{H}^{(2)}$ (depending on $H$ only) such that

$$
\|f\|_{L_{H}^{2}(\mathbb{R})} \leq C_{H}^{(2)}\|f\|_{W_{2}^{2}(\mathbb{R})} .
$$

Proof. According to equality (5.50) of [5],

$$
\left(I_{-}^{\alpha} f\right)(x)=\frac{1}{\Gamma(\alpha+1)} \frac{d}{d x} \int_{0}^{\infty} f(x+u) u^{\alpha} d u=\frac{1}{\Gamma(\alpha+1)} \int_{x}^{\infty} f^{\prime}(u)(u-x)^{\alpha} d u
$$


for functions $f \in C^{1}(\mathbb{R})$ satisfying condition 1$)$. Thus

$$
\begin{aligned}
\|f\|_{L_{H}^{2}(\mathbb{R})} \leq C_{H}^{(3)} & {\left[\left(\int_{\mathbb{R}}\left|\int_{x}^{x+1} f^{\prime}(u)(u-x)^{\alpha} d u\right|^{2} d x\right)^{1 / 2}\right.} \\
& \left.+\left(\int_{\mathbb{R}}\left|\int_{x+1}^{\infty} f^{\prime}(u)(u-x)^{\alpha} d u\right|^{2} d x\right)^{1 / 2}\right],
\end{aligned}
$$

where $C_{H}^{(3)}=C_{H}^{(1)}(\Gamma(\alpha+1))^{-1}$.

Now

$$
\begin{aligned}
& \left(\int_{\mathbb{R}}\left|\int_{x}^{x+1} f^{\prime}(u)(u-x)^{\alpha} d u\right|^{2} d x\right)^{1 / 2} \\
& \quad \leq(2 H)^{-1 / 2}\left(\int_{\mathbb{R}} \int_{x}^{x+1}\left|f^{\prime}(u)\right|^{2} d u d x\right)^{1 / 2}=(2 H)^{-1 / 2}\left\|f^{\prime}\right\|_{L_{2}(\mathbb{R})}
\end{aligned}
$$

and

$$
\begin{aligned}
& \left(\int_{\mathbb{R}}\left|\int_{x+1}^{\infty} f^{\prime}(u)(u-x)^{\alpha} d u\right|^{2} d x\right)^{1 / 2} \\
& \quad=\left(\int_{\mathbb{R}}\left|f(x+1)-\alpha \int_{x+1}^{\infty} f(u)(u-x)^{\alpha-1} d u\right|^{2} d x\right)^{1 / 2} \\
& \quad \leq\|f\|_{L_{2}(\mathbb{R})}+|\alpha|\left(\int_{\mathbb{R}}\left|\int_{x+1}^{\infty} f(u)(u-x)^{\alpha-1} d u\right|^{2} d x\right)^{1 / 2} .
\end{aligned}
$$

An application of the generalized Minkowski inequality yields

$$
\left(\int_{\mathbb{R}}\left|\int_{x+1}^{\infty} f(u)(u-x)^{\alpha-1} d u\right|^{2} d x\right)^{1 / 2} \leq-\frac{1}{\alpha}\|f\|_{L_{2}(\mathbb{R})} .
$$

This means that Theorem 2.1 holds for the constant

$$
C_{H}^{(2)}=C_{H}^{(3)}\left((2 H)^{-1 / 2} \vee 2\right) \text {. }
$$

Remark 2.1. The bound

$$
\mathrm{E}\left|\int_{\mathbb{R}} f(t) d B_{t}^{H}\right|^{2} \leq c_{H}\|f\|_{L_{1 / H}(\mathbb{R})}^{2}
$$

is obtained in the paper [4] for $\int_{\mathbb{R}} f(t) d B_{t}^{H}$ and $H>1 / 2$, where $c_{H}$ is a constant depending on $H$ only. It is also proved in [4 that this bound does not hold for $H<1 / 2$. Instead, a corresponding lower bound

$$
\mathrm{E}\left|\int_{\mathbb{R}} f(t) d B_{t}^{H}\right|^{2} \geq c_{H}\|f\|_{L_{1 / H}(\mathbb{R})}^{2}
$$

is derived in [4] and analogous estimates for $\mathrm{E}\left|\int_{\mathbb{R}} f(t) d B_{t}^{H}\right|^{r}, r>2$, are found.

It follows from Theorem 2.1 that if $H \in(0,1 / 2)$, then the expectation $\mathrm{E}\left|\int_{\mathbb{R}} f(t) d B_{t}^{H}\right|^{2}$ can be estimated for a narrower class of functions of $W_{2}^{2}(\mathbb{R})$ that possess an extra property 1). 
(b) Now let the support of a function $f(x)$ belong to $(0, T)$ for some $T>0$. Then one can get upper moment bounds similar to those obtained in Theorem 2.1 under weaker assumptions posed on the function $f$. In this case,

$$
\left(I_{-}^{\alpha} f\right)(x)=\left(I_{T-}^{\alpha} f\right)(x)= \begin{cases}0, & x \geq T, \\ -\frac{1}{\Gamma(\alpha+1)} \frac{d}{d x} \int_{x}^{T} f(t)(t-x)^{\alpha} d t, & x \in(0, T), \\ \frac{(-\alpha)}{\Gamma(\alpha+1)} \int_{0}^{T} f(t)(t-x)^{\alpha-1} d t, & x \leq 0 .\end{cases}
$$

Consider a particular case of relation (5). Note that

$$
\left(I_{T-}^{\alpha} f\right)(t)=\left(D_{T-}^{-\alpha} f\right)(t)
$$

is the corresponding fractional derivative for $t \in(0, T)$.

Let $f$ belong to the class $I_{T-}^{-\alpha}\left(L_{p}(0, T)\right), p>1$; that is, $f$ can be represented in the form of a fractional integral, namely

$$
f(x)=\frac{1}{\Gamma(-\alpha)} \int_{x}^{T} \varphi(t)(t-x)^{-1-\alpha} d t,
$$

where $\varphi \in L_{p}(0, T)$. According to [5],

$$
\begin{aligned}
& \left(D_{T-}^{-\alpha} f\right)(x) \\
& \quad=\frac{1}{\Gamma(\alpha+1)}\left(f(x)(T-x)^{\alpha}+\alpha \int_{x}^{T}(f(x)-f(t))(t-x)^{\alpha-1} d t\right) 1_{(0, T)}(x) .
\end{aligned}
$$

Remark 2.2. If $f \in C^{\beta}(0, T)$, that is, if $f$ belongs to the Hölder class of order $\beta$ on the interval $(0, T)$, and if $\alpha+\beta>0$, then relation (6) obviously holds. The norm of a function is defined in the space $C^{\beta}(0, T)$ in a standard way, namely

$$
\|f\|_{C^{\beta}(0, T)}=\sup _{0 \leq t \leq T}|f(t)|+\sup _{0 \leq s<t \leq T} \frac{|f(t)-f(s)|}{(t-s)^{\beta}} .
$$

Remark 2.3. According to [5, Theorem 13.4], $f \in I_{T-}^{-\alpha}\left(L_{p}(0, T)\right)$ for some $1<p<\infty$ if and only if $f(x)(T-x)^{\alpha} \in L_{p}(0, T)$ and

$$
\sup _{\varepsilon>0} \int_{0}^{T-\varepsilon}\left|\psi_{\varepsilon}(x)\right|^{p} d x<\infty
$$

where

$$
\psi_{\varepsilon}(x)=\int_{x+\varepsilon}^{T}(f(t)-f(x))(t-x)^{\alpha-1} d t .
$$

In particular, if $f \in I_{T-}^{-\alpha}\left(L_{p}(0, T)\right)$, then $f \in L_{p}(0, T)$. Moreover,

$$
\int_{0}^{T} f(x) d B_{t}^{H}=\int_{\mathbb{R}}\left(M_{-}^{H} f 1_{(0, T)}\right)(t) d W_{t},
$$

where $\left(M_{-}^{H} f 1_{(0, T)}\right)=C_{H}^{(1)}\left(I_{T-}^{\alpha} f\right)(t)$ and $I_{T-}^{\alpha} f$ is defined by (15)-(6). Thus relations (5) (7) imply the following result. 
Lemma 2.1. Let $f \in I_{T-}^{-\alpha}\left(L_{p}(0, T)\right)$; in particular, let $f \in C^{\beta}(0, T)$ and $\alpha+\beta>0$. Then

$$
\begin{aligned}
\left(\mathrm{E}\left|\int_{0}^{T} f(t) d B_{t}^{H}\right|^{2}\right)^{1 / 2} & \left(\left(C_{H}^{(4)}\right)^{2} \int_{-\infty}^{0}\left|\int_{0}^{T} f(t)(t-x)^{\alpha-1} d t\right|^{2} d x\right. \\
= & \left.+\left(C_{H}^{(5)}\right)^{2} \int_{0}^{T}\left|f(x)(T-x)^{\alpha}+\alpha \int_{x}^{T}(f(x)-f(t))(t-x)^{\alpha-1} d t\right|^{2} d x\right)^{1 / 2} \\
\leq & \left.C_{H}^{(4)}\left(\int_{-\infty}^{0}\left|\int_{0}^{T} f(t)(t-x)^{\alpha-1} d t\right|^{2} d x\right)^{1 / 2}\right)^{1 / 2} \\
& +C_{H}^{(5)}\left(\int_{0}^{T}|f(x)|^{2}(T-x)^{2 \alpha} d x\right)^{1 / 2} \\
& +C_{H}^{(5)}\left(|\alpha| \int_{0}^{T}\left(\int_{x}^{T}|f(x)-f(t)|(t-x)^{\alpha-1} d t\right)^{2} d x\right)^{1 / 2}
\end{aligned}
$$

where $C_{H}^{(4)}=-\alpha C_{H}^{(1)} / \Gamma(\alpha+1)$ and $C_{H}^{(5)}=-C_{H}^{(4)} / \alpha$.

Now we estimate the second moment $\mathrm{E}\left|\int_{0}^{T} f(t) d B_{t}^{H}\right|^{2}$ with the help of the norms of the function $f$ in some integral spaces. We assume that the interval $[0, T]$ is fixed. Consider the following classes of functions:

$$
\begin{aligned}
D_{H}(0, T)=\{f:[0, T] & \rightarrow \mathbb{R} \mid \\
\left.\|f\|_{D_{H}(0, T)}^{2}:=\int_{0}^{T}\left(\int_{x}^{T}|f(x)-f(t)|(t-x)^{\alpha-1} d t\right)^{2} d x<\infty\right\}, & \\
L_{H}^{2}(0, T) & =\left\{f:\left.[0, T] \rightarrow \mathbb{R}\left|\int_{0}^{T}\right|\left(M_{-}^{H} f\right)(x)\right|^{2} d x<\infty\right\} .
\end{aligned}
$$

Note that $M_{-}^{H} f=M_{-}^{H}\left(f 1_{(0, T)}\right)$.

Theorem 2.2. $\quad$ (a) For an arbitrary $p>1 / H$, it follows that

$$
I_{T-}^{-\alpha}\left(L_{p}(0, T)\right) \cap D_{H}(0, T) \subset L_{H}^{2}(0, T)
$$

and there exist constants $C_{H}^{(6)}$ and $C_{H}^{(7)}$ such that

$$
\begin{aligned}
\left(\mathrm{E}\left|\int_{0}^{T} f(t) d B_{t}^{H}\right|^{2}\right)^{1 / 2} & \leq C_{H}^{(6)}\|f\|_{L_{p}(0, T)} T^{H-1 / p}+C_{H}^{(7)}\|f\|_{D_{H}(0, T)} \\
& =: G^{1}(0, T, f) .
\end{aligned}
$$

(b) If $\alpha+\beta>0$, then $C^{\beta}(0, T) \subset L_{H}^{2}(0, T)$ and there are constants $C_{H}^{(8)}$ and $C_{H}^{(9)}$ such that

$$
\begin{aligned}
& \left(\mathrm{E}\left|\int_{0}^{T} f(t) d B_{t}^{H}\right|^{2}\right)^{1 / 2} \\
& \quad \leq\|f\|_{C^{\beta}(0, T)}\left(C_{H}^{(8)} T^{H}+C_{H}^{(9)} T^{H+\beta}\right)=: G^{2}(0, T, f) .
\end{aligned}
$$


Proof. (a) We estimate the following integral:

$$
\begin{aligned}
\mathcal{I}_{f}^{1} & :=\int_{0}^{T} \int_{0}^{T}|f(t)||f(v)| \int_{-\infty}^{0}((t-x)(v-x))^{H-3 / 2} d x d t d v \\
& \leq \int_{0}^{T} \int_{0}^{T}|f(t)||f(v)|\left(\int_{-\infty}^{0}(\sqrt{v t}-x)^{2 H-3} d x\right) d t d v \\
& =\frac{1}{2-2 H}\left(\int_{0}^{T}|f(t)| t^{H-1} d t\right)^{2} \\
& \leq C_{1}(H, p)\left(\int_{0}^{T}|f(t)|^{p} d t\right)^{2 / p} T^{(2 H p-2) / p}
\end{aligned}
$$

where $p>1 / H$ and

$$
C_{1}(H, p)=\left(\frac{p-1}{p H-1}\right)^{2(p-1) / p} \frac{1}{2(1-H)} .
$$

Thus $\mathcal{I}_{f}^{1}<\infty$ if $f \in L_{p}(0, T)$. The first integral on the right hand side of (8) is equal to

$$
\mathcal{I}_{f}^{2}:=\int_{0}^{T} \int_{0}^{T} f(t) f(v) \int_{-\infty}^{0}((t-x)(v-x))^{\alpha-1} d x d t d v
$$

by Fubini's theorem and is thus estimated by the right hand side of (11). It is also clear that

$$
\mathcal{I}_{f}^{3}:=\int_{0}^{T}|f(x)|^{2}(T-x)^{2 H-1} d x \leq C_{2}(H, p)\|f\|_{L_{p}(0, T)}^{2} T^{2 H-2 / p}
$$

where $C_{2}(H, p)=((p-2) /(2 H p-2))^{(p-2) / p}$.

This together with (8) implies bound (9) for

$$
C_{H}^{(6)}=C_{H}^{(4)}\left(C_{1}(H, p)\right)^{1 / 2}+C_{H}^{(5)}\left(C_{2}(H, p)\right)^{1 / 2}, \quad C_{H}^{(7)}=C_{H}^{(5)}|\alpha| .
$$

(b) If $f \in C^{\beta}(0, T)$, then

$$
\begin{gathered}
\mathcal{I}_{f}^{2} \leq \frac{1}{2(1-H)}\left(\int_{0}^{T}|f(x)| t^{H-1} d t\right)^{2} \leq \frac{1}{2(1-H) H^{2}}\|f\|_{C^{\beta}(0, T)} T^{2 H}, \\
\mathcal{I}_{f}^{3} \leq \frac{1}{2 H}\|f\|_{C^{\beta}(0, T)} T^{2 H}
\end{gathered}
$$

and

$$
\|f\|_{D_{H}(0, T)}^{2} \leq \frac{1}{2(\alpha+\beta)^{2}(H+\beta)}\|f\|_{C^{\beta}(0, T)}^{2} T^{2 H+2 \beta},
$$

whence bound (10) follows for

$$
\begin{gathered}
C_{H}^{(8)}=C_{H}^{(4)}\left(2(1-H)^{-1 / 2} H^{-1}+C_{H}^{(5)}(2 H)^{-1 / 2}\right), \\
C_{H}^{(9)}=C_{H}^{(5)}|\alpha|(\alpha+\beta)^{-1}(2(H+\beta))^{-1 / 2} .
\end{gathered}
$$

Remark 2.4. Theorem 2.2 shows that an upper bound can be obtained for the second moment $\mathrm{E}\left|\int_{0}^{T} f(t) d B_{t}^{H}\right|^{2}$ if $f \in L_{p}(0, T), p>1 / H$, and $H<1 / 2$. However this bound is expressed in terms of "stronger" norms as compared to the case of $H>1 / 2$ (see the paper [3]). The space $W_{T}^{\beta, \infty}(0, T), 0<\beta<1$, of measurable functions $f:[0, T] \rightarrow \mathbb{R}$ such that

$$
\|f\|_{\beta, \infty, T}:=\sup _{0 \leq s<t \leq T}\left(\frac{|f(t)-f(s)|}{(t-s)^{\beta}}+\int_{s}^{t} \frac{|f(y)-f(s)|}{(y-s)^{1+\beta}} d y\right)<\infty
$$


for some $0<\beta<1$ is considered in the paper [6]. It is mentioned in [6] that

$$
C^{\beta+\varepsilon}(0, T) \subset W_{T}^{\beta, \infty}(0, T) \subset C^{\beta}(0, T)
$$

for all $\varepsilon>0$.

It is obvious that $W_{T}^{-\alpha, \infty}(0, T) \subset D_{H}(0, T)$; hence

$$
I^{-\alpha}\left(L_{p}(0, T)\right) \cap W_{T}^{-\alpha, \infty}(0, T) \subset L_{H}^{2}(0, T)
$$

and bound (9) holds.

Corollary 2.1. Since the integrals $\int_{0}^{t} f(t) d B_{t}^{H}, f \in L_{H}^{2}(0, T)$, are zero mean Gaussian random variables,

$$
\left(\mathrm{E}\left|\int_{0}^{T} f(t) d B_{t}^{H}\right|^{r}\right)^{1 / r} \leq \frac{\sqrt{2}}{\pi^{1 / 2}}\left(\Gamma\left(\frac{r+1}{2}\right)\right)^{1 / r} G^{1}(0, T, f)
$$

for all $r>0$ under assumptions (a). Under assumptions (b), the same inequality holds with $G^{2}(0, T, f)$ instead of $G^{1}(0, T, f)$.

Theorem 2.3 below is similar to Theorem 2.2 The interval $[s, t] \subset[0, T]$ in Theorem 2.3 is arbitrary. Denote by $I^{-\alpha}\left(L_{p}(s, t)\right), p>1$, the class of functions $f$ that can be represented in the form of a fractional integral

$$
f(x)=\frac{1}{\Gamma(1-\alpha)} \int_{x}^{t} \varphi(u)(u-x)^{-1-\alpha} d u, \quad \varphi \in L_{p}(s, t) .
$$

For $0 \leq s<t \leq t_{1} \leq T$, let

$$
\begin{gathered}
D_{H}\left(s, t, t_{1}\right)=\{f:[0, T] \rightarrow \mathbb{R} \mid \\
\left.\|f\|_{D_{H}(s, t)}^{2}=\int_{s}^{t}\left(\int_{x}^{t_{1}}|f(x)-f(u)|(u-x)^{\alpha-1} d u\right)^{2} d x<\infty\right\}, \\
D_{H, p}\left(s, t, t_{1}\right)=\{f:[0, T] \rightarrow \mathbb{R} \mid \\
\left.\|f\|_{D_{H, p}\left(s, t, t_{1}\right)}^{p}=\int_{s}^{t}\left(\int_{x}^{t_{1}}|f(x)-f(u)|(u-x)^{\alpha-1} d u\right)^{p} d x<\infty\right\}, \\
L_{H}^{2}(s, t)=\left\{f:\left.[0, T] \rightarrow \mathbb{R}\left|\int_{s}^{t}\right|\left(M_{-}^{H} f\right)(x)\right|^{2} d x<\infty\right\} .
\end{gathered}
$$

Theorem 2.3. For all $p>1 / H$ and all $0 \leq s<t \leq t_{1} \leq T$, it follows that

$$
\left(L_{p}(s, t)\right) \cap D_{H}\left(s, t, t_{1}\right) \subset L_{H}^{2}(s, t)
$$

and

$$
\begin{aligned}
\left(\mathrm{E}\left|\int_{s}^{t} f(u) d B_{u}^{H}\right|^{2}\right)^{1 / 2} & \leq C_{H}^{(6)}\|f\|_{L_{p}(s, t)} T^{H-1 / p}+C_{H}^{(7)}\|f\|_{D_{H}\left(s, t, t_{1}\right)} \\
& \leq C_{H}^{(6)}\|f\|_{L_{p}(s, t)} T^{H-1 / p}+C_{H}^{(7)}\|f\|_{D_{H, p}\left(s, t, t_{1}\right)} T^{1 / 2-1 / p} \\
& =: G_{p}^{1}\left(s, t, t_{1}, f\right) .
\end{aligned}
$$


Remark 2.5. Let

$$
\begin{gathered}
D_{H, p}(0, T)=\{f:[0, T] \rightarrow \mathbb{R} \mid \\
\left.\|f\|_{D_{H, p}(0, T)}^{p}=\int_{0}^{T}\left(\int_{x}^{T}|f(x)-f(u)|(u-x)^{\alpha-1} d u\right)^{p} d x<\infty\right\}, \\
\quad p>2 .
\end{gathered}
$$

The following bound is similar to (91):

$$
\begin{aligned}
\left(\mathrm{E}\left|\int_{0}^{T} f(u) d B_{u}^{H}\right|^{2}\right)^{1 / 2} & \leq C_{H}^{(6)}\|f\|_{L_{p}(0, T)} T^{H-1 / p}+C_{H}^{(7)}\|f\|_{D_{H, p}(0, T)} T^{1 / 2-1 / p} \\
& =G_{p}^{1}(0, T, T, f) .
\end{aligned}
$$

One can use the entropy estimate for the maximum of a Gaussian stochastic process (see, for example, 7, Theorem 1, Section 13]) to prove maximal moment estimates for Wiener integrals. First we give some other estimates that are useful in other problems, too.

\section{Some maXimal inequalities for Gaussian RANDOM VARIABles AND STOCHASTIC PROCESSES}

Let $r \geq 1$ and let $\psi_{r}(x), x \geq 1$, be a convex function such that $\psi_{r}(x)=(\ln x)^{r / 2}$ for $x \geq \exp (r / 2)$ and $\psi_{r}(x) \geq(\ln x)^{r / 2}$ for $1 \leq x \leq \exp (r / 2)$. For example, one can choose $\psi_{r}(x)=(\ln x)^{r / 2}$ for $1 \leq r \leq 2$ and

$$
\psi_{r}(x)= \begin{cases}(\ln x)^{r / 2}, & x \geq \exp (r / 2), \\ \psi_{r}(x)=c(x-1)^{\beta}, & 1 \leq x \leq \exp (r / 2)\end{cases}
$$

for $r>2$ where $c=(r / 2)^{r / 2}\left(e^{r / 2}-1\right)^{1-e^{-r / 2}}$ and $\beta=1-e^{-r / 2}$.

Lemma 3.1. Let $\left\{\xi_{i}, 1 \leq i \leq N\right\}$ be zero mean Gaussian random variables, that is, $\xi_{i}=N\left(0, \sigma_{i}^{2}\right), \sigma^{2}=\max _{1 \leq i \leq N} \sigma_{i}^{2}>0$, and let $r \geq 1$.

Then

$$
\left(\mathrm{E}\left(\max _{1 \leq i \leq N}\left|\xi_{i}\right|^{r}\right)\right)^{1 / r} \leq Z_{r}(N) \sigma
$$

where

$$
Z_{r}(N)=\inf _{0<s<1}\left(\left(\psi_{r}\left(\frac{N}{\sqrt{1-s}}\right)\right)^{1 / r} \sqrt{\frac{2}{s}}\right)
$$

Proof. Let

$$
\eta:=\max _{1 \leq i \leq N}\left|\xi_{i}\right| .
$$

Then

$$
\begin{aligned}
\mathrm{E}|\eta|^{r}\left(\frac{s}{2 \sigma^{2}}\right)^{r / 2} & =\mathrm{E}\left(\ln \exp \left\{\frac{s \eta^{2}}{2 \sigma^{2}}\right\}\right)^{r / 2} \leq \mathrm{E} \psi_{r}\left(\exp \left\{\frac{s \eta^{2}}{2 \sigma^{2}}\right\}\right) \leq \psi_{r}\left(\mathrm{E} \exp \left\{\frac{s \eta^{2}}{2 \sigma^{2}}\right\}\right) \\
& =\psi_{r}\left(\mathrm{E} \exp \left\{\frac{s}{2 \sigma^{2}} \max _{1 \leq k \leq N} \xi_{k}^{2}\right\}\right) \leq \psi_{r}\left(N \max _{1 \leq k \leq N} \mathrm{E} \exp \left\{\frac{s}{2 \sigma^{2}} \xi_{k}^{2}\right\}\right) \\
& \leq \psi_{r}\left(N \max _{1 \leq k \leq N} \mathrm{E} \exp \left\{\frac{s}{2 \sigma_{k}^{2}} \xi_{k}^{2} 1_{\left\{\sigma_{k} \neq 0\right\}}\right\}\right)=\psi_{r}\left(N \frac{1}{\sqrt{1-s}}\right)
\end{aligned}
$$

whence inequality (13) follows. 
Lemma 3.2. Let random variables $\left\{\xi_{i}, 1 \leq i \leq N\right\}$ be the same as in Lemma 3.1, Then

$$
\left(\mathrm{E} \max _{1 \leq i \leq N}\left|\xi_{i}\right|^{r}\right)^{1 / r} \leq \sigma\left(N\left(C_{r / \alpha}\right)\right)^{\alpha / r}
$$

for $0<\alpha \leq 1$, where

$$
C_{q}=\frac{1}{\sqrt{2 \pi}} \int_{R}|u|^{q} e^{-u^{2} / 2} d u=\frac{2^{q / 2}}{\pi^{1 / 2}} \Gamma\left(\frac{q+1}{2}\right) .
$$

Proof. For $\eta=\max _{1 \leq i \leq N}\left|\xi_{i}\right|$, we get

$$
\begin{aligned}
\mathrm{E}\left(\frac{\eta}{\sigma}\right)^{r} & \leq\left(\mathrm{E}\left(\frac{\eta}{\sigma}\right)^{r / \alpha}\right)^{\alpha} \leq N^{\alpha} \max _{1 \leq i \leq N}\left(\mathrm{E}\left(\frac{\eta_{i}}{\sigma}\right)^{r / \alpha}\right)^{\alpha} \\
& \leq N^{\alpha} \max _{i: \sigma_{i} \neq 0}\left(\mathrm{E}\left(\frac{\eta_{i}}{\sigma_{i}}\right)^{r / \alpha}\right)^{\alpha}=N^{\alpha}\left(C_{r / \alpha}\right)^{\alpha} .
\end{aligned}
$$

Now we apply Lemmas 3.1 and 3.2 to estimate the maximum of a separable Gaussian process.

Let $X=\left\{X_{t}, t \in \mathbb{T}\right\}$ be a Gaussian stochastic process such that $\mathrm{E} X_{t}=0, \mathbb{T}$ an arbitrary set of parameters, and

$$
\rho(s, t):=\left(\mathrm{E}\left|X_{s}-X_{t}\right|^{2}\right)^{1 / 2} .
$$

Denote by $N(u), u>0$, the minimal number of points forming a $u$-set of the space $(\mathbb{T}, \rho)$; that is, $N(u)$ is the minimal number of balls of radius $u$ that cover $\mathbb{T}$. Further let $H(u)=\ln N(u)$ be the metric entropy of the space $(\mathbb{T}, \rho)$ (one can assume that this space is compact), $\|\cdot\|_{r}=\|\cdot\|_{L_{r}(\Omega)}$.

Theorem 3.1. Let the process $X$ assume values in a pseudometric space $(\mathbb{T}, \rho)$ and be separable on it. Then

$$
\left\|\sup _{t \in T}\left|X_{t}\right|\right\|_{r} \leq\left\|X_{t_{0}}\right\|_{r}+\frac{1}{\theta(1-\theta)} \int_{0}^{\varepsilon_{0} \theta} Z_{r}(N(u)) d u
$$

for all $r \geq 1, t_{0} \in \mathbb{T}$, and $0<\theta<1$, where the number $Z_{r}(\cdot)$ is defined by equality (14) and where

$$
\varepsilon_{0}=\sup _{t \in \mathbb{T}} \rho\left(t_{0}, t\right) .
$$

Proof. Let $\varepsilon_{n}=\theta^{n} \varepsilon_{0}, n \geq 1,0<\theta<1$, and let $S_{n}$ be the minimal $\varepsilon_{n}$-net in the space $(\mathbb{T}, \rho)$. Put $S=\bigcup_{n=0}^{\infty} S_{n}$. A result mentioned in [8, p. 102] implies that

$$
\sup _{t \in T}\left|X_{t}\right| \leq\left|X_{t_{0}}\right|+\sum_{n=1}^{\infty} \max _{s \in S_{n}}\left|X_{s}-X_{\alpha_{n-1}(s)}\right|
$$

with probability one for a separable Gaussian stochastic process $X$ where $\alpha_{k}(\cdot)$ is a mapping $\alpha_{k}: S \rightarrow S_{k}$ such that $\alpha_{u}(s)=s$ if $s \in S_{k}$, and $\alpha_{k}(s)$ is a closest point to $s$ if $s \notin S_{k}$ (it does not matter which closest point is chosen if there are several such points). Note that $\rho\left(X_{s}, X_{\alpha_{n-1}(s)}\right) \leq \varepsilon_{n-1}$. It follows from (16) and inequality (14) of Lemma 3.1 
that

$$
\begin{aligned}
\left\|\sup _{t \in T}\left|X_{t}\right|\right\|_{r} & \leq\left\|X_{t_{0}}\right\|_{r}+\sum_{n=1}^{\infty}\left\|\max _{s \in S_{n}}\left|X_{s}-X_{\alpha_{n-1}(s)}\right|\right\|_{r} \\
& \leq\left\|X_{t_{0}}\right\|_{r}+\sum_{n=1}^{\infty} Z_{r}\left(N\left(\varepsilon_{n}\right)\right) \varepsilon_{n-1}=\left\|X_{t_{0}}\right\|_{r}+\sum_{n=1}^{\infty} Z_{r}\left(N\left(\varepsilon_{0} \theta^{n}\right)\right) \varepsilon_{0} \theta^{n-1} \\
& \leq\left\|X_{t_{0}}\right\|_{r}+\frac{1}{\theta(1-\theta)} \sum_{n=1}^{\infty} \int_{\varepsilon_{0} \theta^{n+1}}^{\varepsilon_{0} \theta^{n}} Z_{r}(N(u)) d u \\
& =\left\|X_{t_{0}}\right\|_{r}+\frac{1}{\theta(1-\theta)} \int_{0}^{\varepsilon_{0} \theta} Z_{r}(N(u)) d u
\end{aligned}
$$

Remark 3.1. Note that the integral on the right hand side of (17) is finite,

$$
\int_{0}^{\varepsilon_{0} \theta} Z_{r}(N(u)) d u<\infty
$$

if $\int_{0+}(H(u))^{1 / 2} d u<\infty$, that is, if the Dudley integral $D(\beta):=\int_{0}^{\beta}(H(u))^{1 / 2} d u$ converges for some $\beta>0$ (and therefore for all $\beta>0$ ).

Theorem 3.2. If the assumptions of Theorem 3.1 hold for all $0<\alpha \leq 1$ and $0<\theta<1$, then

$$
\left\|\sup _{t \in T}\left|X_{t}\right|\right\|_{r} \leq\left\|X_{t_{0}}\right\|_{r}+\frac{\left(C_{r / \alpha}\right)^{\alpha / r}}{\theta(1-\theta)} \int_{0}^{\varepsilon_{0} \theta}(N(u))^{\alpha / r} d u .
$$

Proof. Estimate (16) and inequality (15) of Lemma 3.2 imply that

$$
\begin{aligned}
\left\|\sup _{t \in T}\left|X_{t}\right|\right\|_{r} & \leq\left\|X_{t_{0}}\right\|_{r}+\sum_{n=1}^{\infty}\left\|\max _{s \in S_{n}}\left|X_{s}-X_{\alpha_{n-1}(s)}\right|\right\|_{r} \\
& \leq\left\|X_{t_{0}}\right\|_{r}+\sum_{n=1}^{\infty}\left(N\left(\varepsilon_{0} \theta^{n}\right)\right)^{\alpha / r}\left(C_{r / \alpha}\right)^{\alpha / r} \varepsilon_{0} \theta^{n-1} \\
& \leq\left\|X_{t_{0}}\right\|_{r}+\left(C_{r / \alpha}\right)^{\alpha / r} \frac{1}{\theta(1-\theta)} \int_{0}^{\varepsilon_{0} \theta}(N(u))^{\alpha / r} d u .
\end{aligned}
$$

Lemma 3.3. Let $\mathbb{T}=[a, b], b>a$, and let $\rho(s, t)$ be a pseudometric on $\mathbb{T}$. Assume that $\varphi(x), x>0$, is a continuous increasing function such that $\varphi(0)=0$. Let there exist a function $g(u) \geq 0, g \in L_{1}(a, b)$, such that

$$
\varphi(\rho(s, t)) \leq \int_{s}^{t} g(v) d v
$$

for all $a \leq s<t \leq b$. Denote by $N(u), u>0$, the minimal number of closed $(\mathbb{T}, \rho)$ balls of radius $u>0$ that cover $\mathbb{T}$. Then

$$
N(u) \leq 1+\frac{\int_{a}^{b} g(v) d v}{\varphi(2 u)} .
$$

Proof. Let $s_{0}=a$ and $s_{0}<s_{1}<\cdots<s_{M}<b$ where the points $s_{1}, \ldots, s_{M}$ are chosen such that the radius of closed intervals $\left[s_{k}, s_{k+1}\right], k=0, \ldots, M-1$, in the metric $\rho$ equals $u$, while the radius of the interval $\left[s_{M}, b\right]$ does not exceed $u$. Such a partition exists, since the function $\rho(s, t)$ is continuous in $s$ and $t$ according to the assumptions of the lemma. 
It is clear that

$$
\varphi(2 u) \leq \int_{s_{k}}^{s_{k+1}} g(v) d v, \quad k=0, \ldots, M-1,
$$

and $N(u) \leq M+1$.

Thus

$$
M \varphi(2 u) \leq \sum_{k=0}^{M-1} \int_{s_{k}}^{s_{k+1}} g(u) d u=\int_{a}^{s_{M}} g(u) d u \leq \int_{a}^{b} g(u) d u,
$$

that is, $M \leq \int_{a}^{b} g(v) d v / \varphi(2 u)$.

4. UPPER MAXimal BOUND FOR the Wiener INTEGRAL With RESPECT to FRACTIONAL BROWNIAN MOTION

Let

$$
I_{t}=\int_{0}^{t} f(s) d B_{s}^{H}
$$

where $B^{H}=\left\{B_{t}^{H}, t \geq 0\right\}$ is a fractional Brownian motion with Hurst index

$$
H \in(0,1 / 2) .
$$

Applying estimate (9) of Theorem 2.2 to the interval $[0, t]$ and using the notation introduced in Theorem 2.3 we have $f \in L_{H}^{2}(0, t)$ for all $t>0$ if

$$
f \in I^{-\alpha}\left(L_{p}(0, t)\right) \cap D_{H, p}(0, t, t)
$$

for some $p>1 / H$ and all $t \in[0, T]$.

Lemma 4.1. Let condition (20) hold for the stochastic process $\left\{I_{t}, t \geq 0\right\}$ and for all

$$
t \in[0, T] .
$$

Then the trajectories of the separable modification of the process $I_{t}$ are continuous on $[0, T]$ almost surely.

Proof. It is well known that every stochastic process defined on a fixed interval $[0, T]$ has a separable modification. The finiteness of the Dudley integral

$$
D_{T}(\beta)=\int_{0}^{\beta}\left(H_{T}(u)\right)^{1 / 2} d u
$$

is sufficient for the continuity of trajectories of this modification (see [7]), where

$$
H_{T}(u)=\ln N_{T}(u)
$$

and $N_{T}(u)$ is the minimal number of closed intervals of radius $u$ that cover $\mathbb{T}=[0, T]$. The radius of an interval is evaluated with respect to the pseudometric

$$
\rho(s, t)=\left(\mathrm{E}\left(\int_{s}^{t} f(u) d B_{u}^{H}\right)^{2}\right)^{1 / 2} .
$$

Applying estimate (12) of Theorem 2.3 for $t_{1}=T$ we get

$$
\varphi(\rho(s, t)) \leq \int_{s}^{t} g(v) d v
$$

for the functions $\varphi(x)=x^{p}, x \geq 0$, and

$$
g(v)=2^{p-1}\left(\left(C_{H}^{(6)}\right)^{p}|f(v)|^{p} T^{H p-1}+\left(C_{H}^{(7)}\right)^{p}\left(\int_{v}^{T}|f(v)-f(t)|(t-v)^{\alpha-1} d t\right)^{p} T^{p / 2-1}\right) .
$$


Now Lemma 3.3 implies that

$$
N_{T}(u) \leq 1+\int_{0}^{T} g(v) d v \cdot(2 u)^{-p}=1+\int_{0}^{T} \frac{g(v)}{2^{p}} d v \cdot u^{-p}=: 1+G^{3}(0, T, f) u^{-p},
$$

where

$$
\begin{aligned}
G^{3}(0, T, f)=2^{p-1} \int_{0}^{T}\left[\left(C_{H}^{(6)}\right)^{p} \mid\right. & \left.f(v)\right|^{p} T^{p H-1} \\
& \left.+\left(C_{H}^{(7)}\right)^{p}\left(\int_{v}^{T}|f(v)-f(t)|(t-v)^{\alpha-1} d t\right)^{p} T^{p / 2-1}\right] d v
\end{aligned}
$$

Thus

$$
\int_{0}^{\beta}\left(H_{T}(u)\right)^{1 / 2} d u \leq \int_{0}^{\beta}\left(\ln \left(1+G^{3}(0, T, f) u^{-p}\right)\right)^{1 / 2} d u \leq c \int_{0}^{\beta} u^{(-p / 2) \gamma} d u
$$

for small $\beta>0$ and for all $\gamma>0$. Therefore the Dudley integral converges if $\gamma<2 / p$.

Remark 4.1. In what follows we consider a separable modification of $I_{t}=\int_{0}^{t} f(s) d B_{s}^{H}$. This means that trajectories of $I_{t}$ are almost surely continuous on $[0, T]$ by condition (20).

Now we prove maximal moment inequalities.

Theorem 4.1. Let condition (20) hold. Then

$$
\left\|\sup _{0 \leq t \leq T}\left|I_{t}\right|\right\|_{r} \leq G_{r}^{4}(0, T, f):=C_{p, r} G_{p}^{1}(0, T, T, f)
$$

for all $r>0$. One can use the constant $C_{p, r}=2^{3 / 2+1 / 2 p}\left(C_{2 p}\right)^{1 / 2 p}$ for $r<2 p$ or

$$
C_{p, r}=\frac{2^{2+1 / r}}{1-p / r}\left(C_{r}\right)^{1 / r}
$$

for $r \geq 2 p$ in the above inequality. The factor $G_{p}^{1}(0, T, T, f)$ is defined in inequality (12).

Proof. By Lemma 4.1 and Remark 4.1 the process $I_{t}$ is continuous. Estimate (18) of Theorem 3.2 for $t_{0}=0$ and inequality (21) imply that

$$
\left\|\sup _{0 \leq t \leq T}\left|I_{t}\right|\right\|_{r} \leq \frac{\left(C_{r / \alpha}\right)^{\alpha / r}}{\theta(1-\theta)} \int_{0}^{\varepsilon_{0} \theta}\left(1+u^{-p} G^{3}(0, T, f)\right)^{\alpha / r} d u, \quad 0<\alpha \leq 1 .
$$

First we consider the case of $r<2 p$. Put $\alpha=r /(2 p)$. We find $\theta_{0}$ such that

$$
\left(\varepsilon_{0} \theta_{0}\right)^{-p} G^{3}(0, T, f)=1
$$

It is clear that $\theta_{0}=\left(G^{3}(0, T, f)\right)^{1 / p} \varepsilon_{0}^{-1}$, where

$$
\varepsilon_{0}=\sup _{0 \leq t \leq T}\left(\mathrm{E}\left|\int_{0}^{t} f(u) d B_{u}^{H}\right|^{2}\right)^{1 / 2}
$$

By Theorem 2.3.

$$
\varepsilon_{0} \leq G_{p}^{1}(0, T, T, f) \leq 2\left(G^{3}(0, T, f)\right)^{1 / p}
$$

whence we obtain $\theta_{0}>\frac{1}{2}$. Therefore

$$
u^{-p} G^{3}(0, T, f) \geq 1 \quad \text { for } 0<u \leq \varepsilon_{0} / 2
$$


if $\theta=\frac{1}{2}$. Then

$$
\begin{aligned}
\left\|\sup _{0 \leq t \leq T}\left|I_{t}\right|\right\|_{r} & \leq 8\left(C_{2 p}\right)^{2 p} / 2^{1 / 2 p} \int_{0}^{\varepsilon_{0} / 2} u^{-1 / 2} d u\left(G^{3}(0, T, f)\right)^{1 / 2 p} \\
& =2^{3+1 / 2 p}\left(C_{2 p}\right)^{1 / 2 p}\left(G^{3}(0, T, f)\right)^{1 / 2 p}\left(\frac{\varepsilon_{0}}{2}\right)^{1 / 2} \\
& \leq 2^{3 / 2+1 / 2 p}\left(C_{2 p}\right)^{1 / 2 p}\left(G^{3}(0, T, f)\right)^{1 / p} \\
& \leq 2^{3 / 2+1 / 2 p}\left(C_{2 p}\right)^{1 / 2 p} G_{p}^{1}(0, T, T, f) .
\end{aligned}
$$

Now let $r \geq 2 p$. We put $\alpha=1$ and $\theta=1 / 2$ in inequality (18). Then we deduce from (22) that

$$
\begin{aligned}
\left\|\sup _{0 \leq t \leq T}\left|I_{t}\right|\right\|_{r} & \leq 4\left(C_{r}\right)^{1 / r} \int_{0}^{\varepsilon_{0} / 2}\left(1+u^{-p} G^{3}(0, T, f)\right)^{1 / r} d u \\
& \leq 4\left(C_{r}\right)^{1 / r} 2^{1 / r}\left(G^{3}(0, T, f)\right)^{1 / r} \int_{0}^{\varepsilon_{0} / 2} u^{-p / r} d u \\
& =\frac{2^{2+1 / r}}{1-p / r}\left(C_{r}\right)^{1 / r}\left(G^{3}(0, T, f)\right)^{1 / r}\left(\frac{\varepsilon_{0}}{2}\right)^{1-p / r} \\
& \leq \frac{2^{2+1 / r}}{1-p / r}\left(C_{r}\right)^{1 / r}\left(G^{3}(0, T, f)\right)^{1 / p} .
\end{aligned}
$$

The proof of Theorem 4.1 follows from (22) and (24).

Remark 4.2. The following estimate can be found in the book [7]:

$$
\mathrm{P}\left\{\sup _{0 \leq t \leq T}\left|\xi_{t}\right|>\beta\right\} \leq 2\left(1-\Phi\left(\beta-4 \cdot 2^{1 / 2} D_{T}(\sigma / 2)\right) / \sigma\right)
$$

for all $\beta \geq 4 \cdot 2^{1 / 2} D_{T}(\sigma / 2)$ where $\xi$ is a zero mean separable Gaussian stochastic process, $\Phi(\cdot)$ is the standard Gaussian distribution function, and $\sigma^{2}=\sup _{0<t \leq T} \mathrm{E} \xi_{t}^{2}$. Using this estimate one can prove a maximal moment inequality, similar to (22) and (24):

$$
\left\|\sup _{0 \leq t \leq T}\left|I_{t}\right|\right\|_{r} \leq C_{H, r} G^{1}(0, T, T, f),
$$

where

$$
\begin{gathered}
C_{H, r}=\left((4 \sqrt{2})^{r} r^{-r} I_{r}^{r}+r 2^{r} b_{r}+2^{r} r^{2-r}(4 \sqrt{2})^{r-1} b_{1} I_{r}^{r-1}\right)^{1 / r}, \\
I_{r}=\int_{\ln 2}^{\infty} z^{1 / 2} \frac{\exp z d z}{(\exp z-1)^{1+1 / r}} \\
b_{r}=\int_{0}^{\infty} x^{r-1}(1-\Phi(x)) d x=\frac{2^{r / 2-1}}{\pi^{1 / 2}} \Gamma\left(\frac{r+1}{2}\right)
\end{gathered}
$$

(this result for $H>1 / 2$ is obtained in 44). The asymptotic behavior of estimates (24) and (25) is the same as $r \rightarrow \infty$, namely $C_{p, r} \sim C r$ and $C_{H, r} \sim C r$ for some (possibly different) constants $C$. In contrast, estimate (25) holds for Gaussian processes only, while Theorems 3.1, 3.2, and 4.1 can be generalized for the case of processes of the Orlicz spaces of random variables. We plan to prove this generalization in the forthcoming second part of the paper. 


\section{BIBLIOGRAPHY}

1. R. S. Liptser and A. N. Shiryaev, Theory of Martingales, "Nauka", Moscow, 1986; English transl., Kluwer Academic Publishers, Dordrecht, 1989. MR1022664 (90j:60046)

2. C. Bender, Integration with Respect to a Fractional Brownian Motion and Related Market Models, Ph.D. Thesis, Konstanz University, 2003.

3. J. Memin, Yu. Mishura, and E. Valkeila, Inequalities for the moments of Wiener integrals with respect to fractional Brownian motions, Statist. Probab. Lett. 51 (2001), no. 2, 197-206. MR 1822771 (2002b:60096)

4. Yu. V. Krvavich and Yu. S. Mishura, Some maximal inequalities for moments of Wiener integrals with respect to fractional Brownian motion, Teor. Imovir. Mat. Stat. 61 (1999), 72-83; English transl. in Theory Probab. Math. Statist. 61 (2000), 75-86. MR 1866968(2002h:60072)

5. S. G. Samko, A. A. Kilbas, and O. I. Marichev, Fractional Integrals and Derivatives. Theory and Applications, "Nauka i tekhnika", Minsk, 1987; English transl., Gordon and Breach Science Publishers, New York, 1993. MR1347689 (96d:26012)

6. D. Nualart and A. Răşcanu, Differential equations driven by fractional Brownian motion, Collect. Math. 53 (2002), no. 1, 55-81. MR 1893308 (2003f:60105)

7. M. A. Lifshits, Gaussian Random Functions, Kluwer Academic Publishers, Dordrecht, 1995. MR.1472736 (98k:60059)

8. V. V. Buldygin and Yu. V. Kozachenko, Metric Characterization of Random Variables and Random Processes, "TViMS", Kiev, 1998; English transl., AMS, Providence, Rhode Island, 2000. MR.1743716 (2001g:60089)

Department of Probability Theory and Mathematical Statistics, Faculty for Mechanics and Mathematics, National Taras Shevchenko University, Academician Glushkov Avenue, 6 , KYIV 03127, UKRAINE

E-mail address: yvk@univ.kiev.ua

Department of Probability Theory and Mathematical Statistics, Faculty for Mechanics and Mathematics, National Taras Shevchenko University, Academician Glushkov Avenue, 6, KYIV 03127, UKRAINE

E-mail address: myus@univ.kiev.ua

Received 1/DEC/2005

Translated by O. I. KLESOV 